\title{
Exploration of macrophage colony-stimulating factor as a new type of tumor marker
}

\author{
RU ZHOU, YIMING ZHOU and ZONGYOU CHEN \\ Department of General Surgery, Huashan Hospital, Fudan University, Shanghai 200040, P.R. China
}

Received May 14, 2013; Accepted September 18, 2013

DOI: $10.3892 /$ br.2013.170

\begin{abstract}
Gastric cancer is a common type of malignancy with a high incidence of mortality. Therefore, tumor markers should be identified to screen for various types of cancer. Elevated serum concentrations of macrophage colony-stimulating factor (M-CSF) have been found in a variety of malignant diseases. The aim of the present study was to investigate the possibility of serum M-CSF as a new type of tumor marker and to determine its effectiveness when combinedd with other tumor markers. Serum was collected from 32 gastric cancer patients, who were initially diagnosed by gastroscopy, at the Department of General Surgery of Huashan Hospital betwee July, 2010 and December, 2011, and 8 controls. The serum level of M-CSF was measured by enzyme-linked immunosorbent assay kits (ELISA). Clinical and pathological testing was conducted to analyze the differences in the serum level of M-CSF, as comparing to traditional tumor markers. Carcinoembryonic antigen (CEA) and M-CSF levels were found to be significantly higher in the gastric cancer group as compared to the non-gastric cancer group $(\mathrm{P}<0.05)$. CEA levels were significantly elevated when the gastric cancer lesions infiltrated the serosa $(\mathrm{P}=0.046)$. Additionally, the increased levels of M-CSF were of statistical significance when there was lymph node involvement in gastric cancer. For distant metastasis, the levels of M-CSF were decreased $(\mathrm{P}=0.026)$, however, the ratio of CEA to $\mathrm{M}-\mathrm{CSF}$ values increased significantly $(\mathrm{P}=0.048)$. Furthermore, the M-CSF level was positively correlated with TNM stage in gastric cancer patients without distant organ metastasis, in contrast to gastric cancer patients with distant organ metastasis. In conclusion, M-CSF may be considered as a new type of tumor marker that can be combined with traditional tumor markers in order to determine whether the cancer migrated to distant organs.
\end{abstract}

Correspondence to: Professor Zongyou Chen, Department of General Surgery, Huashan Hospital, 12 Middle Wulumuqi Road, Shanghai 200040, P.R. China

E-mail: zhru0456168@gmail.com

Key words: macrophage colony-stimulating factor, gastric cancer, tumor mark, macrophage

\section{Introduction}

Gastric cancer is one of the most common types of cancer worldwide. In China, there is a high morbidity and mortality rate due to atypical symptoms being ignored, resulting in the screening of patients at advanced stage of gastric cancer. Testing for serum tumor markers is convenient and a valid method for screening for different types of cancer. Carcinoembryonic antigen (CEA) and carbohydrate antigen (CA)72-4 are commonly used as tumor markers for gastric cancer, and $\alpha$-fetoprotein (AFP) is used as an indicator to screen for liver metastasis following diagnosis of gastric cancer. Traditional tumor markers are produced by tumor tissues and can indicate the existence and development of tumors. However, more effective tumor markers that can be used to screen for gastric cancer should be identified.

Determining the mechanism behind tumor metastasis is crucial during disease development and progression. The study of tumor immunology, particularly the innate immunity response has demonstrated that $(1,2)$ the initial step of tumor metastasis, i.e., released tumor cells invading the matrix, then entering the vascular system, cannot be considered as the rate-limiting step of metastasis. Findings of previous studies have demonstrated that in the early stages of tumorigenesis, a high number of tumor cells are detected in the circulatory system, while cell content in the circulatory system does not correlate with tumor stage. Since more tumor cells may be present in early stage tumors in the circulatory system as compared to more advanced stage tumors, distant metastasis is more likely to occur. Therefore, metastasis should not be regarded as the end-point as of the thousands of circulating cells released by tumors only a few successfully result in metastasis. In their study, Joyce and Pollard (1) demonstrated that extravasation and establishment of micro-metastasis were major rate-limiting events.

Macrophages are known to populate metastatic lesions (1), however, focus on their role in metastasis is a recent event. Macrophage is an important member of the innate immune system and plays an important role during the process of tumor progression through tumor cell shedding, invasive growth, entering into circulation, formation of metastasis sites and formation of the nutrient vessels. CSF is an important factors, whose function is the recruitment of macrophages.

Involvement of macrophage-associated colony-stimulating factors in the process of tumor metastasis is common in a 
variety of neoplastic diseases. A Polish study (2) demonstrated serum macrophage colony-stimulating factor (M-CSF) levels in patients with colorectal cancer to be higher as compared to the control groups, with correlations being identified between $\mathrm{M}-\mathrm{CSF}$ and lymph node metastasis and tumor stage. The level of M-CSF decreased following radical surgery, as did CEA. $\mathrm{M}-\mathrm{CSF}$ is also thought to be a factor indicating prognosis. Furthermore, there have been similar findings concerning CSF in studies on esophageal cancer (3), multiple myeloma (4), pancreatic cancer (5), prostate cancer (6), gynecologic cancer (7), breast cancer (8) and head and neck cancer (9). When the expression of CSF in vivo increases, it often indicates that the tumor is more invasive, more prone to distant metastasis and overall prognosis is poor. This study was conducted to investigate the suitability of M-CSF as a novel tumor marker in combination with existing tumor markers.

\section{Materials and methods}

Patients. The study included 32 previously untreated gastric cancer patients (Table I) who were diagnosed and treated at the General Surgery Department at Huashan Hospital, Fudan University, between July, 2010 and December, 2011. Eight patients suffering from other gastric diseases (4 women and 4 men, aged 57-68 years) including lymphoma, gastrointestinal stromal tumor, retroperitoneal tumor and anastomotic polyps following subtotal gastrectomy were considered the control group. None of the patients had received chemo- or radiotherapy prior to blood sample collection. A clinical diagnosis of gastric cancer was confirmed each time by microscopic examination of the material obtained during endoscopy, biopsy and/or surgery. Gastric cancers were histologically verified as adenocarcinomas. During surgery, radical lymph node dissection was uniformly performed. Postoperative, pathological staging (primary tumor, regional lymph node involvement and the occurrence of distant metastasis) was performed by correlating the surgical and histological findings. The tumors were staged in accordance with the standard of National Comprehensive Cancer Network gastric cancer guideline (10). To determine TNM staging as well as whether distant organ metastasis occurred, patients were divided into groups. The study was approved by the local ethics committee of Huashan Hospital, Fudan University (Shanghai, China). Institutional review board approval was obtained and informed consent from all participants.

Data of the serum levels of traditional tumor markers were obtained from the Department of Laboratory Medicine of Huashan Hospital, and were tested when patients were admitted to the hospital. The cut-off values of traditional tumor markers in our hospital are: CEA, $10 \mu \mathrm{g} / 1$; CA19-9, $37 \mu \mathrm{g} / \mathrm{l}$; AFP, $10 \mu \mathrm{g} / \mathrm{l}$; CA72-4, $8.2 \mu \mathrm{g} / 1$. The pathology reports were issued by the Department of Pathology of Huashan Hospital.

Blood samples and assays. Venous blood samples were collected from each patient on admission, centrifuged to obtain serum samples and stored at $-80^{\circ} \mathrm{C}$ until assayed. Tested cytokine was measured using enzyme-linked immunosorbent assay kits (ELISA) (CK-E10133H; R\&D Systems, Minneapolis, $\mathrm{MN}$, USA) according to the manufacturer's instructions.
Table I. Characteristics of gastric cancer patients.

\begin{tabular}{lcc}
\hline Parameter & $\begin{array}{c}\text { No. of } \\
\text { patients }\end{array}$ & Percentage \\
\hline Age (years) & & \\
59.4 (average) & 32 & 100 \\
28-90 (range) & 32 & 100 \\
Gender & & \\
Men & 18 & 56.2 \\
Women & 14 & 43.8 \\
Bowel wall invasion (T stage) & & \\
Tis & 3 & 9.4 \\
T1 & 5 & 15.6 \\
T2 & 3 & 9.4 \\
T3 & 13 & 40.6 \\
T4 & 8 & 25 \\
Lymph node metastasis (N stage) & & \\
N0 & 11 & 34.4 \\
N1 & 5 & 15.6 \\
N2 & 3 & 9.4 \\
N3 & 6 & 18.7 \\
Nx & 7 & 21.9 \\
Distant metastasis (M stage) & & \\
M0 & 28 & 87.5 \\
M1 & 4 & 12.5 \\
UICC TNM stage & 4 & \\
0 & & \\
I & & \\
II & 5.4 \\
III & & 12.5 \\
IV & 5 & \\
\hline
\end{tabular}

UICC, Union for International Cancer Control.

Statistical analysis. The measurement data were described by using the mean \pm standard deviation. For comparisons between two groups fitting normal distribution the Student's t-test was used and while the $\chi^{2}$ test was used to compare between groups. $\mathrm{P}<0.05$ was considered to indicate a statistically significant difference. Analyses were conducted using the SPSS (version 19.0) program (IBM, Armonk, NY, USA).

\section{Results}

Diagnosis in this study was confirmed by the pathology results combined with imaging and clinical manifestations. The efficiency of various tumor markers and their combination were calculated based on the diagnosis (Table II). The results showed that CEA and CA19-9 were the most effective tumor markers for the diagnosis of gastric cancer as compared to CA72-4 in terms of specificity, whereas the combination of all tumor markers was most effective with regard to sensitivity.

Gastric cancer patients were divided into groups according to the different T, N, M and TNM staging, as well as the average 
Table II. The efficiency of traditional tumor markers in the diagnosis of gastric cancer ${ }^{\mathrm{a}}$.

\begin{tabular}{lrcc}
\hline Tumor markers & $\begin{array}{c}\text { Sensitivity } \\
(\%)\end{array}$ & $\begin{array}{c}\text { Specificity } \\
(\%)\end{array}$ & $\begin{array}{c}\text { Accuracy } \\
(\%)\end{array}$ \\
\hline CEA & 19.4 & 100 & 32.4 \\
CA19-9 & 9.6 & 100 & 24.3 \\
CA72-4 & 20.8 & 66.7 & 25.9 \\
AFP & 6.9 & 83.3 & 20.0 \\
CEA+CA72-4 & 29.0 & 83.3 & 37.8 \\
All of above & 32.3 & 50.0 & 35.1 \\
\hline
\end{tabular}

aGastric cancer group, 32 patients; non-gastric cancer group, 8 patients. CEA, carcinoembryonic antigen; CA, carbohydrate antigen; AFP, $\alpha$-fetoprotein.

Table III. Serum levels of M-CSF and tumor markers in patients with or without gastric cancer.

\begin{tabular}{lccc}
\hline Variables & $\begin{array}{c}\text { Gastric } \\
\text { cancer group }\end{array}$ & $\begin{array}{c}\text { Non-gastric } \\
\text { cancer group }\end{array}$ & P-value \\
\hline No. of patients & 32 & 8 & - \\
M-CSF $(\mu \mathrm{g} / 1)$ & $0.80 \pm 1.27$ & $0.31 \pm 0.06$ & $0.018^{\mathrm{a}}$ \\
$\mathrm{CEA}(\mu \mathrm{g} / \mathrm{l})$ & $5.76 \pm 9.40$ & $2.04 \pm 1.12$ & $0.022^{\mathrm{a}}$ \\
$\mathrm{AFP}(\mu \mathrm{g} / \mathrm{l})$ & $37.92 \pm 166.9$ & $13.41 \pm 24.89$ & 0.23 \\
$\mathrm{CA72-4}(\mu \mathrm{g} / \mathrm{l})$ & $16.21 \pm 49.65$ & $47.58 \pm 58.28$ & 0.26 \\
\hline
\end{tabular}

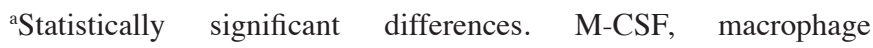
colony-stimulating factor; CEA, carcinoembryonic antigen; AFP, $\alpha$-fetoprotein; CA, carbohydrate antigen.

of the indices was calculated in each group (Tables III-VI). The results demonstrated that serum levels of CEA and M-CSF were significantly higher in gastric cancer patients as compared to non-gastric cancer patients.

We found that only the serum CEA showed a significantly higher expression with tumor invasion of the serosa. CEA was also able to distinguish late T stages (Table IV).

Based on the incidence of lymph node metastasis, gastric cancer patients were divided into two groups (Table V). Only the expression of M-CSF was increased when lymph node metastasis occurred. Thus, only M-CSF is an indicator of lymph node metastasis for gastric cancer patients.

The results showed that only four cases of gastric cancer patients were stage IV. However, during the follow-up, another two cases developed lesions of hepatic metastasis $<3$ months after radical gastrectomy (D2 lymph node dissection). As a result, six cases of gastric cancer patients were classified in the group 'Simultaneous metastasis' (Table VI). Additionally, although the levels of individual traditional tumor markers increased but were not statistically significant, the levels of M-CSF decrease and statistical significance was observed.
Table IV. Various indicators in gastric cancer patients with local early or late stage.

\begin{tabular}{lccc}
\hline T stage & Tis/T1/T2 & T3/T4 & P-value \\
\hline No. of patients & 11 & 21 & - \\
M-CSF $(\mu \mathrm{g} / 1)$ & $0.49 \pm 0.53$ & $0.96 \pm 1.49$ & 0.21 \\
CEA $(\mu \mathrm{g} / 1)$ & $2.20 \pm 1.56$ & $7.72 \pm 11.17$ & $0.046^{\mathrm{a}}$ \\
AFP $(\mu \mathrm{g} / \mathrm{l})$ & $3.32 \pm 2.08$ & $56.12 \pm 204.81$ & 0.28 \\
CA72-4 $(\mu \mathrm{g} / \mathrm{l})$ & $1.99 \pm 1.05$ & $22.06 \pm 57.99$ & 0.18 \\
\hline
\end{tabular}

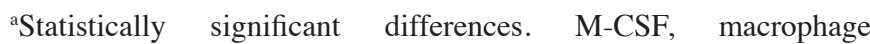
colony-stimulating factor; CEA, carcinoembryonic antigen; AFP, $\alpha$-fetoprotein; CA, carbohydrate antigen.

Table V. Indicators of gastric cancer patients with or without lymph nodes metastasis.

\begin{tabular}{lccc}
\hline N stage & N negative & N positive & P-value \\
\hline No. of patients & 15 & 17 & - \\
M-CSF $(\mu \mathrm{g} / 1)$ & $0.33 \pm 0.12$ & $1.02 \pm 1.38$ & $0.045^{\mathrm{a}}$ \\
CEA $(\mu \mathrm{g} / \mathrm{l})$ & $2.24 \pm 1.94$ & $5.04 \pm 5.39$ & 0.051 \\
AFP $(\mu \mathrm{g} / \mathrm{l})$ & $2.98 \pm 1.58$ & $2.74 \pm 1.69$ & 0.37 \\
CA72-4 $(\mu \mathrm{g} / \mathrm{l})$ & $3.77 \pm 4.13$ & $8.33 \pm 10.10$ & 0.12 \\
\hline
\end{tabular}

aStatistically significant differences. M-CSF, macrophage colony-stimulating factor; CEA, carcinoembryonic antigen; AFP, $\alpha$-fetoprotein; CA, carbohydrate antigen.

Table VI. Indicators of gastric cancer patients with or without distant organ metastasis.

\begin{tabular}{lccc}
\hline M stage & $\begin{array}{c}\text { Non- } \\
\text { metastasis }\end{array}$ & $\begin{array}{c}\text { Simultaneous } \\
\text { metastasis }\end{array}$ & P-value \\
\hline No. of patients & 26 & 6 & - \\
M-CSF $(\mu \mathrm{g} / \mathrm{l})$ & $0.94 \pm 1.40$ & $0.33 \pm 0.07$ & $0.043^{\mathrm{a}}$ \\
$\mathrm{CEA}(\mu \mathrm{g} / \mathrm{l})$ & $3.08 \pm 3.88$ & $16.96 \pm 15.45$ & 0.05 \\
$\mathrm{AFP}(\mu \mathrm{g} / \mathrm{l})$ & $7.39 \pm 21.96$ & $154.92 \pm 339.83$ & 0.19 \\
$\mathrm{CA72-4}(\mu \mathrm{g} / \mathrm{l})$ & $18.45 \pm 55.55$ & $7.69 \pm 4.14$ & 0.21 \\
CEA/M-CSF ratio & $6.20 \pm 8.07$ & $53.13 \pm 51.55$ & $0.048^{\mathrm{a}}$ \\
\hline
\end{tabular}

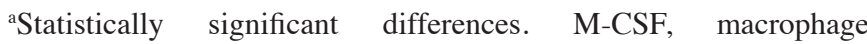
colony-stimulating factor; CEA, carcinoembryonic antigen; AFP, $\alpha$-fetoprotein; CA, carbohydrate antigen.

\section{Discussion}

Diagnosis of gastric cancer was based on the pathological results (Table I), and results of the fitting distributions suggested that locally advanced gastric cancer accounted for the majority of cases while the early stage gastric cancer accounted for the minority of cases. In addition, since the 


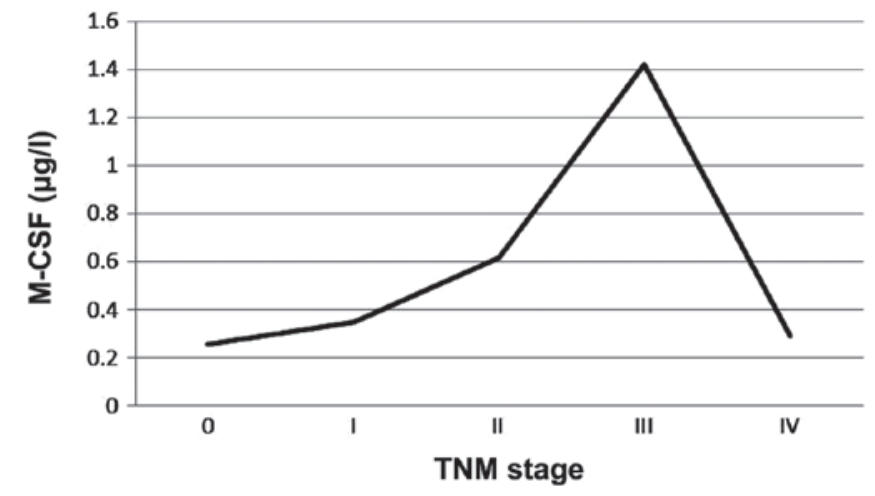

Figure 1. Levels of serum macrophage colony-stimulating factor (M-CSF) in different TNM stages.

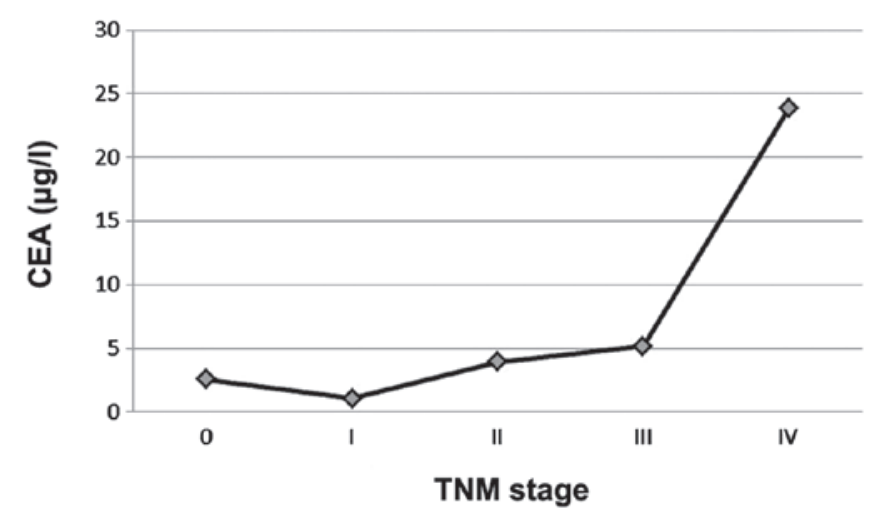

Figure 2. Levels of serum carcinoembryonic antigen (CEA) in different TNM stages.

sample size was insufficient, the diagnosis efficacy of the traditional tumor markers showed less significance (Table II). Due to the lack of standard reference values from the normal population, the diagnostic efficiency of M-CSF was not evaluated.

From the detection of various markers from gastric and non-gastric cancer patients (Table III), the serum levels of CEA and M-CSF from gastric cancer patients were significantly higher compared to the non-gastric cancer group.

We detected indicators in different TNM stages of gastric cancer patients. Prior to stage IV, M-CSF levels increased regularly with staging, although the levels of traditional tumor markers fluctuated. However, at stage IV, M-CSF concentration markedly decreased to a level similar to that of stage I, whereas the levels of the traditional tumor markers increased. CEA and M-CSF were used as examples to demonstrate TNM staging (Figs. 1 and 2).

Findings of a study (11) conduced in Brazil identified serum and intraoperative peritoneal lavage fluid levels of CEA and CA72-4 in patients with gastric cancer. Authors of that study found that the serum level of CA72-4 was the most sensitive tumor marker for gastric cancer and the levels of CEA and CA72-4 in peritoneal lavage fluid were able to distinguish between gastric cancer patients and control patients. Moreover, particularly CEA was capable of distinguishing late $\mathrm{T}$ stages (stage III/IV). Results of this study also presented a similar value of serum CEA to that study (Table IV).
Recent studies have investigated which conventional tumor markers lead to lymph node metastasis in gastric cancer. In a study conducted in Turkey, 75 cases of resectable gastric cancer patients were identified. The patient preoperative serum CA19-9 levels correlated with lymph node metastasis, vascular invasion, cancer stage and tumor size (12). Zhu et al (13) reviewed the preoperative levels of serum tumor markers from 160 cases of gastric cancer patients (Beijing Cancer Hospital) during the years 2002-2007. Their results demonstrated that CEA levels exhibited an improved response in patients with lymph node metastasis as compared to other tumor markers. Moreover, CA72-4 was highly expressed in patients with advanced gastric cancer and vascular invasion.

In the present study, we analyzed various indicators of $\mathrm{N}^{+} / \mathrm{N}^{-}$stage groups (Table $\mathrm{V}$ ). Only M-CSF proved to be a potential indicator suggesting $\mathrm{N}$ positivity and is therefore the only marker capable of determining lymph node metastasis in gastric cancer patients.

The gastric cancer patients were divided into two groups according to whether or not there was distant organ metastasis (Table VI). Although the levels of traditional tumor markers increased individually and significantly, the difference was not statistically significant. By contrast, the decreased level of M-CSF showed statistical significance.

As traditional tumor markers are secreted by tumor cells, their serum levels increased when the amount of cancer cells in vivo increased, indicating disease progression (Fig. 1). Thus, higher serum levels of tumor markers often indicate worse tumor staging, a greater incidence of lymph nodes and distant metastasis, relatively poorer tumor differentiation, a greater likelihood of tumor recurrence following surgery, even worse overall prognosis. Results of this study demonstrate that increased serum levels of M-CSF together with gastric cancer progression occurred, when there was no distant metastasis. However, when distant organ metastasis occurred (similar to TNM stage IV), the level of M-CSF markedly decreased, abnormally and significantly (Table VI and Fig. 2). Tumor immunology theory can be used to explain this phenomenon, because macrophages are involved when the tumor cells develop into distant metastases, also known as the formation of premetastasis niche (14), when TNM stage IV has not yet been achieved. Currently, millions of macrophages are mobilized systemically, thus the serum levels of M-CSF, known as signal to recruit macrophages, are also the highest levels. When distant metastasis has been formed (TNM stage IV), there is no need to recruit a large number of macrophages, thus there is a decrease in the level of M-CSF.

Based on the abovementioned data, we calculated the ratio of serum level CEA and M-CSF to determine whether there was a correlation with metastasis (Table VI), and a statistically significant difference was identified. It was found that the higher the ratio, the more likely it was for the distant metastasis to occur.

Therefore, M-CSF may serve as a tumor marker. It is different from traditional tumor markers as it is not produced by tumor cells themselves. Unlike traditional tumor markers, the higher serum level of M-CSF did not lead to end stage and the worst prognosis in the present study. Additionally, although the serum levels revealed late tumor stage, no distant metastasis occurred. 
If the aforementioned results are confirmed, M-CSF is likely to become a novel tumor marker that may be used by clinicians to detect gastric cancer in patients as the higher the M-CSF level was, the more likely radical surgery was to be used. Moreover, these findings suggest the potential clinical use of M-CSF measurements, particularly in estimating prognosis for patients with gastric cancer.

\section{References}

1. Joyce JA and Pollard JW: Microenvironmental regulation of metastasis. Nat Rev Cancer 9: 239-252, 2009.

2. Mroczko B, Groblewska M, Wereszczynska-Siemiatkowska U, Okulczyk B, Kedra B, Laszewicz W, Dabrowski A and Szmitkowski M: Serum macrophage-colony stimulating factor levels in colorectal cancer patients correlate with lymph node metastasis and poor prognosis. Clin Chim Acta 380: 208-212, 2007.

3. Lukaszewicz-Zajac M, Mroczko B, Kozlowski M, Niklinski J, Laudanski J and Szmitkowski M: Clinical significance of serum macrophage-colony stimulating factor (M-CSF) in esophageal cancer patients and its comparison with classical tumor markers. Clin Chem Lab Med 48: 1467-1473, 2010.

4. Kowalska M, Kaminska J, Fuksiewicz M, Kotowicz B, Chechlinska M, Druzd-Sitek A and Walewski J: A survey of prognostic value of serum factors in multiple myeloma patients before treatment: macrophage-colony stimulating factor (M-CSF) is a powerful predictor of survival. Med Oncol 28: 194-198, 2011.

5. Groblewska M, Mroczko B, Wereszczynska-Siemiatkowska U, Mysliwiec P, Kedra B and Szmitkowski M: Serum levels of granulocyte colony-stimulating factor (G-CSF) and macrophage colony-stimulating factor (M-CSF) in pancreatic cancer patients. Clin Chem Lab Med 45: 30-34, 2007.
6. Ide H, Hatake K, Terado Y, Tsukino H, Okegawa T, Nutahara K, Higashihara E and Horie S: Serum level of colony-stimulating factor 1 as a marker of tumor progression in prostate cancer patients. In: Ide Hisamitsu. Proceedings of the American Association for Cancer Research Annual Meeting[C](96th Annual Meeting of the American Association for Cancer Research, Anaheim, CA, USA, 2005), Tokyo, Japan: Kyorin Universiy, 47: 747, 2005.

7. Suzuki M, Ohwada M, Sato I and Nagatomo M: Serum level of macrophage colony-stimulating factor as a marker for gynecologic malignancies. Oncology 52: 128-133, 1995.

8. Lawicki S, Omyla J, Mroczko B, Szmitkowski M and Czygier M: Plasma level of macrophage-colony stimulating factor (M-CSF) in the course of breast cancer treatment. Pol Arch Med Wewn 112: 1181-1187, 2004 (In Polish)

9. Kuropkat C, Duenne AA, Herz U, Renz H and Werner JA: Significant correlation of matrix metalloproteinase and macrophage colony-stimulating factor serum concentrations in patients with head and neck cancer. Neoplasma 51: 375-378, 2004.

10. National Comprehensive Cancer Network: Gastric Cancer version v.1.2011. http://www.nccn.org/professionals/physician gls/pdf/gastric.pdf. Accessed .

11. Mandorwski S, Lourenco LG and Forones NM: CA72-4 and CEA in serum and peritoneal washing in gastric cancer. Arq Gastroenterol 39: 17-21, 2002 (In Portuguese).

12. Dilege E, Mihmanli M, Demir U, Ozer K, Bostanci O, Kaya C, Aksakal O and Sakiz D: Prognostic value of preoperative CEA And CA 19-9 levels in resectable gastric cancer. Hepatogastroenterology 57: 674-677, 2010.

13. Zhu YB, Ge SH, Zhang LH, Wang XH, Xing XF, Du H, Hu Y, Li YA, Jia YN, Lin Y, et al: Clinical value of serum CEA, CA19-9, CA72-4 and CA242 in the diagnosis and prognosis of gastric cancer. Zhonghua Wei Chang Wai Ke Za Zhi 15: 161-164, 2012 (In Chinese).

14. Qian BZ and Pollard JW: Macrophage diversity enhances tumor progression and metastasis. Cell 141: 39-51, 2010. 